\title{
General Relativity as a Collection of Collections of Models*
}

\author{
JB Manchak
}

\section{Introduction}

Consider the collection $\mathscr{U}$ of smooth, four-dimensional (connected, Hausdorff) Lorentzian manifolds $\left(M, g_{a b}\right)$. One usually identifies this collection of geometric objects with the models of general relativity (Wald 1984). But within $\mathscr{U}$ lurk 'physically unreasonable' models of spacetime. For example, take any $\left(M, g_{a b}\right) \in$ $\mathscr{U}$ and remove a point $p \in M$. The resulting structure $\left(M-\{p\}, g_{a b}\right) \in \mathscr{U}$ seems to be 'physically unreasonable' in the sense that it is not 'as large as it can be' (see Earman 1995). How can such examples be ruled out? Let us say that a model $\left(M, g_{a b}\right) \in \mathscr{U}$ is inextendible if it cannot be properly and isometrically embedded into some other model $\left(M^{\prime}, g_{a b}^{\prime}\right) \in \mathscr{U}$. (It is immediate that the mutilated example from above is not inextendible since it can be properly and isometrically embedded back into the model we started with.) It has been suggested that we limit the number of 'physically unreasonable' models in general relativity by requiring inextendibility to be satisfied (Geroch 1970).

Other examples could be constructed which are 'physically unreasonable' in other senses. Stepping back, the general suggestion seems to be that, for a variety of collections $\mathscr{P} \subset \mathscr{U}$, one could "modify general relativity as follows: the new theory is to be general relativity, but with the additional condition that only $[\mathscr{P}]$ space-times are permitted" (Geroch 1977, 87). Let us take this idea seriously in what follows. For each 'physically reasonable' collection of models $\mathscr{P} \subset \mathscr{U}$, we have a variant theory of general relativity - call it $\operatorname{GR}(\mathscr{P})$. Since we have yet to identify a privileged collection $\mathscr{P} \subset \mathscr{U}$ of 'physically reasonable' models, it is helpful to think of 'general relativity' in a pluralistic way; we can study a collection of such 'physically reasonable' collections of models. What do we know about the these non-standard formulations of general relativity? In this paper, I work to get a better grip on this situation.

After some preliminaries, the paper is divided into three main parts. In the first part, I consider the 'modal' properties of spacetime. These are properties

\footnotetext{
* This is a draft of a paper to appear in Hajnal Andréka and István Németi on unity of science: from computing to relativity theory through algebraic logic, Judit Madarász and Gergely Székely (eds.), Outstanding Contributions to Logic, Springer. Thanks to David Malament for helping to improve a previous draft. My gratitude to Thomas Barrett, Erik Curiel, Martin Lesourd, David Malament, and Jim Weatherall for helpful discussions on this topic.
} 
- like inextendibility - which are defined relative to a background 'physical possibility space' in the form of some collection of models. Such properties seem to 'work differently' in some of the variant theories of general relativity; in the case of inextendibility, the very same model of spacetime can be considered 'inextendible' according to one variant theory and 'extendible' according to another. Given this sensitivity to theory choice, I argue that an exploration of the modal properties of spacetime among variant theories of general relativity would seem to be quite appropriate.

In the second part of the paper, I focus attention on the property of inextendibility. Consider $\operatorname{GR}(\mathscr{P})$ for some 'physically reasonable' collection $\mathscr{P} \subset$ $\mathscr{U}$. I investigate two questions of interest. First, does the property of inextendibility in $\operatorname{GR}(\mathscr{P})$ 'work the same' (in the sense mentioned above) as it does in $\operatorname{GR}(\mathscr{U})$ ? Second, does every model in $\operatorname{GR}(\mathscr{P})$ have an 'inextendible extension' like it does in GR( $\mathscr{U})$ ? Regarding the first question, I settle some precise questions posed by Geroch (1970) and generalize the results somewhat; the emerging picture seems to be that the property of inextendibility is not well-understood outside of the standard theory. Regarding the second question, I consider a class of variant theories defined using 'local' properties (e.g. the standard energy conditions) and show that members of this class always return an affirmative answer. Similar conclusions can be found with respect to a few other variant theories of interest. But I stress that things can also go the other way and provide an example concerning a 'singularity' of certain type.

In the third part of the paper, I focus attention on the singular structure of spacetime. Following an argument by Geroch (1970), I show a sense in which the definition of a 'singular' spacetime depends on the standard definition of inextendibility. A question naturally presents itself: given a 'physically reasonable' collection of models $\mathscr{P} \subset \mathscr{U}$, what is a singularity in $\operatorname{GR}(\mathscr{P})$ ? I draw attention to the fact that the answer is not so clear in some cases. I conclude the paper with a few remarks concerning the scope of the approach to general relativity considered in this paper. In particular, I highlight the fact that other physical theories can be treated in the same way.

\section{Preliminaries}

We begin with a few preliminaries concerning the relevant background formalism. ${ }^{1}$ An $n$-dimensional, general relativistic model of spacetime (for $n \geq 2$ ) is a pair of mathematical objects $\left(M, g_{a b}\right)$ where $M$ is a smooth, connected, $n$ dimensional, Hausdorff manifold (without boundary) and $g_{a b}$ is a smooth metric of Lorentz signature $(-,+, \ldots,+)$ defined on $M$. We say the models $\left(M, g_{a b}\right)$ and $\left(M^{\prime}, g_{a b}^{\prime}\right)$ are isometric if there is a diffeomorphism $\varphi: M \rightarrow M^{\prime}$ such that $\varphi_{*} g_{a b}=g_{a b}^{\prime}$. The models $\left(M, g_{a b}\right)$ and $\left(M^{\prime}, g_{a b}^{\prime}\right)$ are locally isometric if, for each point $p \in M$, there is an open neighborhood $O$ of $p$ and an open subset $O^{\prime}$ of

\footnotetext{
${ }^{1}$ The reader is encouraged to consult Hawking and Ellis (1973), Wald (1984), and Malament (2012) for details. Less technical surveys of the global structure of spacetime are given by Geroch and Horowitz (1979) and Manchak (2013).
} 
$M^{\prime}$ such that $O$ and $O^{\prime}$ are isometric, and, correspondingly, with the roles of $\left(M, g_{a b}\right)$ and $\left(M^{\prime}, g_{a b}^{\prime}\right)$ interchanged.

A model $\left(M, g_{a b}\right)$ is extendible if there is a model $\left(M^{\prime}, g_{a b}^{\prime}\right)$ and a proper isometric embedding $\varphi: M \rightarrow M^{\prime}$. Here, the model $\left(M^{\prime}, g_{a b}^{\prime}\right)$ is an extension of $\left(M, g_{a b}\right)$. A model is inextendible if it has no extension. We say $\left(M, g_{a b}, F\right)$ is an $n$-dimensional framed model if $\left(M, g_{a b}\right)$ is an $n$-dimensional model and $F$ is an orthonormal $n$-ad of vectors $\left\{\xi_{1}, \ldots, \xi_{n}\right\}$ at some point $p \in M$. We say an $n$-dimensional framed model $\left(M^{\prime}, g_{a b}^{\prime}, F^{\prime}\right)$ is a framed extension of the $n$ dimensional framed model $\left(M, g_{a b}, F\right)$ if there is a proper isometric embedding $\varphi: M \rightarrow M^{\prime}$ which takes $F$ into $F^{\prime}$.

Fix a model $\left(M, g_{a b}\right)$. For each point $p \in M$, the metric assigns a cone structure to the tangent space $M_{p}$. Any tangent vector $\xi^{a}$ in $M_{p}$ will be timelike if $g_{a b} \xi^{a} \xi^{b}<0$, null if $g_{a b} \xi^{a} \xi^{b}=0$, or spacelike if $g_{a b} \xi^{a} \xi^{b}>0$. Null vectors create the cone structure; timelike vectors fall inside the cone while spacelike vectors fall outside. A time orientable model is one that has a continuous timelike vector field on $M$. In what follows, we assume that models are time orientable and that an orientation has been chosen.

For some connected interval $I \subset \mathbb{R}$, a smooth curve $\gamma: I \rightarrow M$ is timelike if its tangent vector $\xi^{a}$ at each point in $\gamma[I]$ is timelike. Similarly, a curve is null if its tangent vector at each point is null. A curve is causal if its tangent vector at each point is either null or timelike. A causal curve is future-directed if its tangent vector at each point falls in or on the future lobe of the light cone. A causal curve $\gamma: I \rightarrow M$ is closed if the tangent vector is nowhere vanishing and there are distinct $s, s^{\prime} \in I$ such that $\gamma(s)=\gamma\left(s^{\prime}\right)$. $\left(M, g_{a b}\right)$ satisfies chronology if it does not contain a closed timelike curve; it satisfies causality if it does not contain a closed causal curve.

We write $p \ll q$ (respectively, $p<q$ ) if there exists a future-directed timelike (respectively, causal) curve from $p$ to $q$. For any point $p \in M$, we define the timelike future of $p$, as the set $I^{+}(p)=\{q: p \ll q\}$. Similarly, the causal future of $p$ is the set $J^{+}(p)=\{q: p<q\}$. The timelike and causal pasts of $p$, denoted $I^{-}(p)$ and $J^{-}(p)$, are defined analogously. $\left(M, g_{a b}\right)$ satisfies distinguishability if there do not exist distinct points $p, q \in M$ such that $I^{-}(p)=I^{-}(q)$ or $I^{+}(p)=I^{+}(q)$. It satisfies global hyperbolicity if it is causal and for any points $p, q \in M$, the set $J^{+}(p) \cap J^{-}(q)$ is compact. ${ }^{2}$

A curve $\gamma: I \rightarrow M$ is maximal if there is no curve $\gamma^{\prime}: I^{\prime} \rightarrow M$ such that $I$ is a proper subset of $I^{\prime}$ and $\gamma(s)=\gamma^{\prime}(s)$ for all $s \in I$. The curve $\gamma: I \rightarrow M$ is a geodesic if $\xi^{a} \nabla_{a} \xi^{b}=\mathbf{0}$ where $\xi^{a}$ is its tangent vector and $\nabla_{a}$ is the unique derivative operator compatible with $g_{a b}$. A maximal geodesic $\gamma: I \rightarrow M$ is incomplete if $I \neq \mathbb{R}$. A model is geodesically incomplete if it harbors an incomplete geodesic and geodesically complete otherwise. A futuredirected causal geodesic $\gamma: I \rightarrow M$ is past-incomplete if it is maximal and there is an $r \in \mathbb{R}$ such that $r<s$ for all $s \in I$.

\footnotetext{
2If one replaces 'causal' by 'strongly causal' one obtains the standard formulation of global hyperbolicity. One can show that the two formulations are equivalent (Bernal, A., and Sánchez, M. (2007).
} 
Let the energy-momentum tensor $T_{a b}$ for the model $\left(M, g_{a b}\right)$ be defined by Einstein's equation: $R_{a b}-\frac{1}{2} R g_{a b}=8 \pi T_{a b}$ where $R_{a b}$ is the Ricci tensor and $R$ the scalar curvature associated with $g_{a b}$. We say that $\left(M, g_{a b}\right)$ is a vacuum solution if $T_{a b}=\mathbf{0}$. The null energy condition is satisfied if, for any null vector $\chi^{a}$, we have $T_{a b} \chi^{a} \chi^{b} \geq 0$. The weak energy condition is satisfied if, for each timelike vector $\xi^{a}$, we have $T_{a b} \xi^{a} \xi^{b} \geq 0$. The strong energy condition is satisfied if, for any unit timelike vector $\xi^{a}$, we have $\left(T_{a b}-\frac{1}{2} T g_{a b}\right) \xi^{a} \xi^{b} \geq 0$. Finally, the dominant energy condition is satisfied if, for any future-directed unit timelike $\xi^{a}$, the vector $T_{b}^{a} \xi^{b}$ is causal and future-directed.

Let $S$ be a set. A relation $\leq$ on $S$ is a partial order if, for all $a, b, c \in S$ : (i) $a \leq a$, (ii) if $a \leq b$ and $b \leq c$, then $a \leq c$, and (iii) if $a \leq b$ and $b \leq a$, then $a=b$. If $\leq$ is a partial ordering on a set $S$, we say a subset $T \subset S$ is totally ordered if, for all $a, b \in T$, either $a \leq b$ or $b \leq a$. Let $\leq$ be a partial ordering on $S$ and let $T \subset S$ be totally ordered. An upper bound for $T$ is an element $u \in S$ such that for all $a \in T, a \leq u$. A maximal element of $S$ is an element $m \in S$ such that for all $c \in S$, if $m \leq c$, then $c=m$. Zorn's lemma (equivalent to the axiom of choice) is the following: Let $\leq$ be a partial order on $S$. If each totally ordered subset $T \subset S$ has an upper bound, there is a maximal element of $S$.

\section{Possibility}

One can think of general relativity as a collection of possible models of spacetime. But what counts as 'possible' in this context? As mentioned above, the collection $\mathscr{U}$ seems to be 'too big' in the sense that some of the geometric possibilities it permits do not appear to be genuine physical possibilities. So one works to pare down the space by examining the "pathological situations with a view toward deciding which situations are to be ruled out by physical considerations" (Geroch 1971, 72). Let us consider two influential suggestions along these lines. As mentioned above, it has been proposed that

$(\dagger)$ "any [physically] reasonable space-time should be inextendible" (Clarke 1993, 8).

The recommendation is that we remove from the background possibility space $\mathscr{U}$ all of the models which are extendible. Indeed, not doing so leads to a situation in which extendible models count as "counter-examples to seemingly plausible [physical] conjectures" (Clarke 1976,17). An analogous position arises with many other properties of interest. For example, consider one version of the (strong) cosmic censorship conjecture (Penrose 1979) which states that

$(\dagger \dagger)$ all "physically reasonable spacetimes are globally hyperbolic" (Wald 1984, 304).

Now suppose we accept both $(\dagger)$ and $(\dagger \dagger)$; suppose we decide to rule out all models which are either extendible or non-globally hyperbolic (or both) from 
our background possibility space $\mathscr{U}$. It is not clear how this is to be done. Let me explain. On the one hand, it seems natural to begin with the collection $(I) \subset \mathscr{U}$ of inextendible models and then pare down this space by removing from it all non-globally hyperbolic models. The resulting space is $(I) \cap(G H)$ where $(G H) \subset \mathscr{U}$ is the collection of globally hyperbolic models. On the other hand, one could begin with the collection of globally hyperbolic models $(G H)$ and work to pare down this space by removing all of the extendible models from it. But here is where some tension arises: we cannot simply identify the resulting sub-collection with $(I) \cap(G H)$ as before and, at the same time, respect the intuitive motivation for the definition of inextendibility. To see the point, consider the 'bottom half' of Misner spacetime. ${ }^{3}$ One can show that the model is globally hyperbolic and extendible, and yet it cannot be extended and remain globally hyperbolic (Chrusciel and Isenberg 1993). So we have a model in $(G H)$ which is 'as large as it can be' in the sense that it cannot be be reasonably extended; and yet it does not make it into the collection $(I) \cap(G H)$.

The tension at issue here can be stated like so: (i) inextendibility is a modal property which has been defined relative to the background possibility space $\mathscr{U}$ and yet (ii) the collection $\mathscr{U}$ does not properly capture the notions of physical possibility we are after. It seems clear to me that if we are to rule out 'physically unreasonable' models of spacetime, then we ought to rule out 'physically unreasonable' definitions of inextendibility as well. Following Geroch (1970) let us say that for any collection $\mathscr{P} \subset \mathscr{U}$, a model is a $\mathscr{P}$-model if it is in the collection $\mathscr{P}$. A $\mathscr{P}$-model is $\mathscr{P}$-extendible if it has an extension in $\mathscr{P}$ (such an extension is a $\mathscr{P}$-extension) and $\mathscr{P}$-inextendible otherwise. We are now in a position to see clearly how it is that inextendibility 'works differently' in some variant theories of general relativity; the truncated Misner example from above is $(G H)$-inextendible but $\mathscr{U}$-extendible.

Given this sensitivity to theory choice, it seems appropriate to study the property of inextendibility from within each 'physically reasonable' variant theory of general relativity. That is, it would seem appropriate to study $\mathscr{P}$ inextendibily for some collection of 'physically reasonable' collections $\mathscr{P} \subset \mathscr{U}$. More on this below. At this stage, we stress that there are a number of other spacetime properties which depend, like inextendibility, on a background 'physical possibility space' in the form of some collection of models. Some of these require spacetime to be 'as large as it can be' in various senses; the properties of 'hole-freeness' and 'local inextendibility' are two examples along these lines in addition to inextendibility. ${ }^{4}$ Properties concerning 'stability' are often modal as well; the condition of 'stable causality' is one such example. ${ }^{5}$ For various collec-

\footnotetext{
${ }^{3}$ See Hakwing and Ellis (1973) or the proof of Proposition 1 below for a precise definition.

${ }^{4}$ See Geroch (1977) and Hawking and Ellis (1973) for introductions to such concepts. See Minguzzi (2012), and Manchak (2014) for discussions and updated definitions.

${ }^{5}$ Some care is required to see this fact. The original definition of stable causality given in (Hawking 1969) is clearly modal. But in practice, a non-modal 'stand in' definition is often used instead (see Wald 1984, 198). The two definitions - along with a third (non-modal) definition concerning the existence of a 'global time function' - are all equivalent in GR( $\mathscr{U})$. But in some variant theories of general relativity, the modal and non-modal properties can come apart.
} 
tions $\mathscr{P} \subset \mathscr{U}$ of 'physically reasonable' models, one could make a study of the properties of ' $\mathscr{P}$-hole-freeness', ' $\mathscr{P}$-local inextendibility', ' $\mathscr{P}$-stable causality', and so on. The associated theorems concerning such ' $\mathscr{P}$ properties' amount to what I will call the 'modal structure' of $\operatorname{GR}(\mathscr{P})$.

Because of (ii) above, we see that a focused study of the modal structure of GR $(\mathscr{U})$ will be insufficient to properly understand the role of physical possibility within general relativity. And it does not seem appropriate to restrict attention to just one variant theory of general relativity either, given that we have yet to pin down - and arguably cannot pin down - a privileged collection of 'physically reasonable' models (Manchak 2011). The situation suggests a natural pluralistic position: to better understand the 'modal structure' of spacetime, we ought to study a collection of collections of 'physically reasonable' models. ${ }^{6}$

\section{Inextendibility}

One response to the discussion above goes something like this: Perhaps for some suitably chosen collection $\mathscr{P} \subset \mathscr{U}$ of 'physically reasonable' models, the modal structures of $\operatorname{GR}(\mathscr{U})$ and $\operatorname{GR}(\mathscr{P})$ will be 'close enough' for all practical purposes. In what follows, I will push back on this line of reasoning. We begin with a question posed by Geroch (1970) concerning inextendibility: For which collections $\mathscr{P} \subset \mathscr{U}$ of 'physically reasonable' models does $\mathscr{P}$-inextendibility 'work the same' as inextendibility? In other words, for which such collections $\mathscr{P} \subset \mathscr{U}$ is the following true?

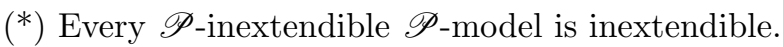

We see that if $\left(^{*}\right)$ is true for some collection $\mathscr{P} \subset \mathscr{U}$, then it makes no difference whether one uses $\mathscr{P}$-inextendibility or the standard definition; any $\mathscr{P}$ model will be $\mathscr{P}$-inextendible if and only if it inextendible. It is of some interest that we have yet to identify a (non-trivial) collection $\mathscr{P}$ of 'physically reasonable' models which renders $\left(^{*}\right)$ true. ${ }^{7}$ In fact there is a growing collection of collections $\mathscr{P} \subset \mathscr{U}$ for which $\left(^{*}\right)$ is known to be false. Here we present a synopsis of the situation so far and draw attention to open questions of significant interest. All proofs for new results can be found in the appendix.

Let (Dist), (Caus), $(C h r) \subset \mathscr{U}$ be the collections of distinguishing, causal, and chronological models respectively and recall that $(G H)$ is the collection of globally hyperbolic models. Basic results concerning the 'causal hierarchy' of spacetime require that $(G H) \subset($ Dist $) \subset($ Caus $) \subset(C h r)$ (Hawking and Ellis 1973). We know from the truncated Misner example from above that $\left(^{*}\right)$ is false

\footnotetext{
${ }^{6}$ One would also like to identify theorems which hold across all variant theories of general relativity. One can think of the logical study of general relativity carried out by Andréka et al. (2010) as one research program along these lines; the first order axioms that are used cannot make reference to a background collection of models.

${ }^{7}$ It is trivial that if one takes $\mathscr{P}$ to be the collection of inextendible models or any subcollection of that collection (e.g. the geodesically complete models), then (*) is true for $\mathscr{P}$.
} 
when $\mathscr{P}=(G H)$. But even more can be said.

Proposition 1. (*) is false for all $\mathscr{P}$ such that $(G H) \subset \mathscr{P} \subset($ Dist $)$.

Proposition 2. (*) is false if $\mathscr{P}=($ Caus $)$.

As a corollary to the first proposition above, we see that $\left(^{*}\right)$ is false for collections $\mathscr{P} \subset \mathscr{U}$ defined relative to a number of other frequently used causal conditions including 'strong causality' and 'stable causality' (see Wald 1984). Another remark may be helpful here; there is no guarantee that, just because $\left({ }^{*}\right)$ is false for $($ Dist $)$ and $($ Caus $)$ and (Dist) $\subset($ Caus $)$, that $\left(^{*}\right)$ must also be false for all $\mathscr{P}$ such that $($ Dist $) \subset \mathscr{P} \subset($ Caus $)$. Each such collection $\mathscr{P}$ must be either checked independently or some new argument must be introduced for why this is not needed. As far as I know, the question of whether $\left(^{*}\right)$ is false if $\mathscr{P}=(C h r)$ is still open (Geroch 1970).

We now turn to the collection $(G I) \subset \mathscr{U}$ of geodesically incomplete models. Presumably the singularity theorems of Hawking and Penrose (1970) show a sense in which (at least some) 'physically reasonable' models can be geodesically incomplete. But it is not difficult to show the following.

Proposition 3. $\left(^{*}\right)$ is false if $\mathscr{P}=(G I)$.

Finally, let us consider some of the 'local' properties of spacetime. Let $(V) \subset \mathscr{U}$ be the collection of vacuum solutions and let $(D E C),(S E C),(W E C)$, $(N E C) \subset \mathscr{U}$ be the collections of models satisfying the dominant, strong, weak, and null energy conditions respectively. Standard results show that $(V) \subset(D E C) \subset(W E C) \subset(N E C)$ and $(V) \subset(S E C) \subset(N E C)$ (Curiel 2017). The question of whether any of these collections satisfy $\left(^{*}\right)$ was asked by Geroch (1970). Concerning $(V)$, things are still open as far as I know. If $\mathscr{P}=(W E C)$, we know that $\left(^{*}\right)$ is false (Manchak 2017). It turns out that the result can be generalized to include all of the standard energy conditions.

Proposition 4. $\left(^{*}\right)$ is false for all $\mathscr{P}$ such that $(D E C) \subset \mathscr{P} \subset(N E C)$. Proposition 5. (*) is false for all $\mathscr{P}$ such that $(S E C) \subset \mathscr{P} \subset(N E C)$.

The emerging picture here seems to be that the property of inextendibility 'works differently' in a now substantial collection of collections $\mathscr{P} \subset \mathscr{U}$ of 'physically reasonable' models. So the modal structure of spacetime does not seem to be well-understood outside of the standard theory $\operatorname{GR}(\mathscr{U}){ }^{8}$

The state of affairs suggests that we explore foundational claims other than $\left(^{*}\right)$ in the manner outlined above. Along these lines, consider that in the standard theory every extendible model has an inextendible extension (Geroch

\footnotetext{
${ }^{8}$ Things are beginning to change on this front, however. See Galloway and Ling (2017) and Sbierski (2018) for work concerning $\mathscr{P}$-inextendibility in cases where where $\mathscr{U} \subset \mathscr{P}$ rather than $\mathscr{P} \subset \mathscr{U}$. See also some recent work on 'time machines' which involves a study of $\mathscr{P}$-inextendibility (Earman et. al 2016; Krasnikov 2018).
} 
1970). ${ }^{9}$ Upon this theorem rests a hefty philosophical weight. For one thing, it underpins the metaphysical justification for the $(\dagger)$ position above (Earman 1995, Manchak 2017). For another, an influential definition of a 'singular' spacetime seems to depend on it (Geroch 1970). We will return to the topic of singularities soon. For now, let us ask: for which collections $\mathscr{P} \subset \mathscr{U}$ of 'physically reasonable' models is the following statement true?

$\left.{ }^{* *}\right)$ Every $\mathscr{P}$-extendible $\mathscr{P}$-model has a $\mathscr{P}$-inextendible $\mathscr{P}$-extension.

It turns out $\left({ }^{* *}\right)$ is true for all 'local' properties of a certain kind. Following Geroch (1970) and Krasnikov (2014), let us say that a property $\mathscr{P} \subset \mathscr{U}$ is local if for each model $\left(M, g_{a b}\right) \in \mathscr{U}$ and any open cover $\left\{V_{\alpha}\right\}$ of $M$, we have: $\left(M, g_{a b}\right) \in \mathscr{P} \Leftrightarrow\left(V_{\alpha}, g_{a b}\right) \in \mathscr{P}$ for all $V_{\alpha} \cdot{ }^{10}$ We have the following.

Proposition 6. $\left.{ }^{* *}\right)$ is true for all local $\mathscr{P} \subset \mathscr{U}$.

It should be clear that, as a corollary to this proposition, $\left({ }^{*}\right)$ is true if $\mathscr{P}$ is any one of the following collections: $(V),(D E C),(S E C),(W E C)$, or $(N E C)$. A few non-local properties also render $(* *)$ true. ${ }^{11}$ In particular, we have the following (see Manchak (2017) for a proof of the first of these results).

Proposition 7. (**) is true if $\mathscr{P}=(C h r)$.

Proposition 8. $\left(^{* *}\right)$ is true if $\mathscr{P}=(G I)$.

One might be tempted to conclude from all of this that $(* *)$ will be true for any collection of 'physically reasonable' models. But consider the collection $(B) \subset(G I)$ of models with the property that every maximal timelike geodesic is past-incomplete. We have the following (Manchak 2016).

Proposition 9. $\left.{ }^{* *}\right)$ is false if $\mathscr{P}=(B)$.

Presumably, the 'big bang' in our own universe permits us to view this property as a 'physically reasonable' one. ${ }^{12}$ In any case, the point here is simply "to demonstrate by some example that a certain assertion is false, or that a certain line of argument cannot work" (Geroch and Horowitz 1979, 221). In the present case, I only wish to stress that it is not yet clear that (**) will be true

\footnotetext{
${ }^{9}$ It is of some interest that this result seems to be one of the few in general relativity to depend crucially on the axiom of choice (in the form of Zorn's lemma) for its proof. See Clarke (1976) for a nice discussion concerning the relationship between set theory and general relativity.

${ }^{10}$ The definition here differs from the one given in (Manchak 2009). One can show that any local property as defined here will be a local property by the lights of (Manchak 2009). It is an open question as to whether the definitions are equivalent.

${ }^{11}$ See Low (2012) for a discussion concerning some of the obstacles preventing even more results of this kind from being established.

${ }^{12} \mathrm{On}$ the other hand, one might argue that quantum considerations drastically change our understanding of the 'big bang' singularity. See Ashtekar (2010) for a discussion.
} 
for all collections $\mathscr{P} \subset \mathscr{U}$ of 'physically reasonable' models. One would like to see a further investigation $\left({ }^{* *}\right)$ with respect to other sub-collections of $(G I)$. In particular, let $(S) \subset(G I)$ be a collection of models satisfying the assumptions of any one of the standard singularity theorems (Hawking and Penrose 1970). One wonders whether $\left({ }^{* *}\right)$ is false if $\mathscr{P}=(S)$. More on singularities and their connection to both $(*)$ and $(* *)$ below.

\section{$5 \quad$ Singularities}

What is a 'singularity' in general relativity? This is a subtle question and a great deal of ink has been spilled in the attempt to find a suitable definition. ${ }^{13}$ But the community seems to have settled on the (non-modal) property of geodesic incompleteness (or some closely related variant) as an imperfect but suitable working 'stand in' for this definition (Wald 1984). Here I would like to draw attention to one sense in which the plausibility of such a position seems to rest on the standard definition of inextendibility. Here is the argument (Geroch 1970, 261-262).

There are two distinct notions to be combined into the definition of a singular spacetime. Let us consider as an example the spacetime $M$ consisting of a small open neighborhood of one of the homogeneous spacelike sections in a Friedmann model. Our $M$ is not 'singular' in the usual intuitive sense (e.g. no scalar invariants become infinite). We would, nonetheless, like to rule out $\mathrm{M}$ as a model of the universe on the grounds that $M$ represents only 'part of the universe'. It is because $\mathrm{M}$ is extendible that the singularities - which are certainly present in the Friedmann models - do not show up in $M$. The first step, then, is to recognize the extendible spacetimes. Suppose now that we have a spacetime $M^{\prime}$ which is inextendible (e.g. the full Friedmann model). We then require a second criterion to recognize that $M^{\prime}$ is 'singular'. The important point is that we do not expect at this stage to be able to recognize singularities in an extendible spacetime because singularities always appear 'at the edge' of the spacetime manifold, and it is precisely this 'edge' which may be missing from an extendible space. That is, we need only formulate a definition of 'singular' which is applicable to inextendible spacetimes. Finally, we may try to generalize our definition to extendible spaces. Thus, we envisage three steps in the definition: (1) define 'extendible', (2) define 'singular' for inextendible spacetimes, and (3) define 'singular' for all spacetimes. (Only the second of these steps is difficult.)

\footnotetext{
${ }^{13}$ See Geroch $(1968 ; 1970)$ and Ellis and Schmidt (1977) for exemplary discussions on the topic. See Weatherall (2014) for recent work on the analogous question concerning geometrized newtonian gravitation.
} 
Geroch goes on to construct a definition of singular spacetime. For step (1), the standard definition of inextendibility is used. In the 'difficult' step (2), we seem to be led to the following imperfect but still suitable definition: an inextendible model is singular ${ }^{\star}$ if it is geodesically incomplete. One reason the definition seems to work is that it allows us to be sure that the geodesic incompleteness present in a singular ${ }^{\star}$ model is not due to its extendibility - one can show that any extendible spacetime will have incomplete causal geodesics (see Clarke 1993, 8). In the final step (3), the definition is generalized: an arbitrary model is singular if (i) it is inextendible and singular ${ }^{\star}$ or (ii) it is extendible and all of its inextendible extensions are singular ${ }^{\star}$.

Clearly this definition of a singular spacetime depends on the standard definition of inextendibility. Given that we have reason to doubt the physical significance of the standard definition of inextendibility, where does this leave us? It would seem that, for each 'physically reasonable' collection $\mathscr{P} \subset \mathscr{U}$, one could straightforwardly define a type of ' $\mathscr{P}$-singular' property. Here, we draw attention to a pair of obstacles in doing so; it is of some interest that the two obstacles correspond to $(*)$ and $(* *)$ from above.

Fix some collection $\mathscr{P} \subset \mathscr{U}$ of 'physically reasonable' models. Carrying out the analogous step (1) is not difficult; one can use the definition of $\mathscr{P}_{-}$ inextendibility. One might be tempted to carry out an analogous step (2) in the following way: a $\mathscr{P}$-inextendible $\mathscr{P}$-model is $\mathscr{P}_{\text {-singular }}{ }^{\star}$ if it is geodesically incomplete. If $(*)$ is true for $\mathscr{P}$, there is no problem. But suppose that $\left(^{*}\right)$ is false for $\mathscr{P}$. In this case, we cannot rule out the possibility that a $\mathscr{P}$-singular ${ }^{\star}$ model is extendible. There seem to be two ways of looking at the situation. ${ }^{14}$ Consider first an extendible $\mathscr{P}$-inextendible model which is such that no scalar invariants become infinite (as is the case for a number examples given in the appendix). Such a model seems to be "not 'singular' in the usual intuitive sense" (Geroch 1970, 261). On the other hand, we know that any extendible spacetime will have incomplete causal geodesics. And it would seem there is "a serious physical pathology" in any such spacetime since "it is possible for at least one freely falling particle or photon to end its existence within a finite 'time'...or to have begun its existence a finite time ago" (Wald 1984, 216). If such a pathology is present, the moniker 'singular' does not seem inappropriate - especially in a model which is 'as large as it can be' in the sense of $\mathscr{P}$-inextendibility.

Suppose the second option is favored and one adopts the definition of $\mathscr{P}_{-}$ singular $^{\star}$. Complications also arise in carrying out the analogous step (3). If $\left({ }^{*}\right)$ is true for $\mathscr{P}$, we can define an arbitrary $\mathscr{P}$-model as $\mathscr{P}$-singular if (i) it is $\mathscr{P}$-inextendible and $\mathscr{P}_{\text {-singular }}^{\star}$ or (ii) it is $\mathscr{P}$-extendible and all of its $\mathscr{P}$ inextendible $\mathscr{P}$-extensions are $\mathscr{P}_{\text {-singular }}^{\star}$ (at least one such extension must exist since $\left({ }^{* *}\right)$ is true for $\left.\mathscr{P}\right)$. But if $\left({ }^{* *}\right)$ is false for $\mathscr{P}$, then there do not exist $\mathscr{P}$-inextendible $\mathscr{P}$-extensions to some $\mathscr{P}$-models and it is not clear how to work around such situations. The upshot seems to be that the question 'what is a singularity in general relativity?' is even more subtle than we might have thought.

\footnotetext{
${ }^{14}$ Thanks to Jim Weatherall for the point.
} 


\section{Conclusion}

Stepping back, we see that the approach to general relativity outlined above can also be implemented with respect to other physical theories. As long as one has a collection of models representing physical possibilities, and as long as there are some models in the collection which are thought to be 'physically unreasonable' in some sense, then an exploration of some collection of collections of 'physically reasonable' models would seem to be fitting. ${ }^{15}$

\section{Appendix}

Proposition 1. $\left(^{*}\right)$ is false for all $\mathscr{P}$ such that $(G H) \subset \mathscr{P} \subset($ Dist $)$.

Proof. Let $\mathscr{P}$ be such that $(G H) \subset \mathscr{P} \subset($ Dist $)$. Consider the Misner model $\left(N, g_{a b}\right)$. Here $N=\mathbb{R} \times S$ and $g_{a b}=2 \nabla_{(a} t \nabla_{b)} \varphi-t \nabla_{a} \varphi \nabla_{b} \varphi$ where the points $(t, \varphi)$ are identified with the points $(t, \varphi+2 \pi n)$ for all integers $n$. Now, let $M=\{(t, \varphi) \in N: t<0\}$ and consider the extendible model $\left(M, g_{a b}\right)$. We know this model is globally hyperbolic (Chrusciel and Isenberg 1993) and therefore in $\mathscr{P}$. We need only show that all of its extensions fail to be in $\mathscr{P}$. Let $\left(M^{\prime}, g_{a b}^{\prime}\right)$ be any extension of $\left(M, g_{a b}\right)$ and let $p$ be a point in $\partial M \cap M^{\prime}$. In any neighborhood of $p$, there will be a point $q \in \partial M \cap M^{\prime}$ such that $q \neq p$. One can verify that $I^{-}(p)=M=I^{-}(q)$. We see that $\left(M^{\prime}, g_{a b}^{\prime}\right)$ is not distinguishing and therefore not in $\mathscr{P}$.

Proposition 2. $\left(^{*}\right)$ is false if $\mathscr{P}=($ Caus $)$.

Proof. Let $\left(M, g_{a b}\right)$ be defined such that $M=\mathbb{R} \times S$ and $g_{a b}=\nabla_{(a} t \nabla_{b)} \varphi-$ $\sinh t^{2} \nabla_{a} \varphi \nabla_{b} \varphi$ where the points $(t, \varphi)$ are identified with the points $(t, \varphi+2 \pi n)$ for all integers $n$ (cf. Malament 2012,135). One can verify that the closed curve at $t=0$ is causal since $(\partial / \partial \varphi)^{a}$ is null there. Moreover, the model was chosen so that the closed causal curve at $t=0$ is the only closed causal curve present. Now consider the model $\left(M^{\prime}, g_{a b}\right)$ where $M^{\prime}=M-\{0,0\}$. It is causal - the 'missing point' prohibits the causal curve at $t=0$ from being closed. But the model has only one extension: the acausal $\left(M, g_{a b}\right)$.

Proposition 3. $\left(^{*}\right)$ is false if $\mathscr{P}=(G I)$.

Proof. Let $\left(M, g_{a b}\right)$ be any geodesically complete model. Now consider the model $\left(M-\{p\}, g_{a b}\right)$ for any point $p \in M$. It is geodesically incomplete because it is extendible. But the model has only one extension: the geodesically complete $\left(M, g_{a b}\right)$.

\footnotetext{
${ }^{15}$ Indeed, Geroch (1977) explicitly considers such an approach with respect to a version of Newtonian spacetime. See also the related discussion in Norton (2003) and Malament (2008).
} 
Lemma 1. Let $\left(\mathbb{R}^{4}, \eta_{a b}\right)$ be Minkowski spacetime where $\eta_{a b}=-\nabla_{a} t \nabla_{b} t+$ $\nabla_{a} x \nabla_{b} x+\nabla_{a} y \nabla_{b} y+\nabla_{a} z \nabla_{b} z$. Let $f: \mathbb{R} \rightarrow \mathbb{R}$ be any smooth function and let $\Omega: \mathbb{R}^{4} \rightarrow \mathbb{R}$ be defined by $\Omega(t, x, y, z)=\exp [f(t)]$. Under Einstein's equation, the energy-momentum tensor associated with $g_{a b}=\Omega^{2} \eta_{a b}$ is given by $T_{a b}=(\rho+p) \nabla_{a} t \nabla_{b} t+p \eta_{a b}$ where $\rho=3 f^{\prime}(t)^{2} / 8 \pi$ and $p=-\left(f^{\prime}(t)^{2}+2 f^{\prime \prime}(t)\right) / 8 \pi$.

Proof. Let $\left(\mathbb{R}^{4}, \eta_{a b}\right)$ be Minkowski spacetime where $\eta_{a b}=-\nabla_{a} t \nabla_{b} t+\nabla_{a} x \nabla_{b} x+$ $\nabla_{a} y \nabla_{b} y+\nabla_{a} z \nabla_{b} z$. Let $f: \mathbb{R} \rightarrow \mathbb{R}$ be any smooth function and let $\Omega: \mathbb{R}^{4} \rightarrow \mathbb{R}$ be defined by $\Omega(t, x, y, z)=\exp (f(t))$. Associated with $g_{a b}=\Omega^{2} \eta_{a b}$ we have the following (Wald 1984, 446).

$$
\begin{aligned}
R_{a b}= & -2 \nabla_{a} \nabla_{b} f(t)-\eta_{a b} \eta^{c d} \nabla_{c} \nabla_{d} f(t)+2\left(\nabla_{a} f(t)\right)\left(\nabla_{b} f(t)\right) \\
& -2 \eta_{a b} \eta^{c d}\left(\nabla_{c} f(t)\right)\left(\nabla_{d} f(t)\right) \\
R= & \Omega^{-2}\left[-6 \eta^{a b} \nabla_{a} \nabla_{b} f(t)-6 \eta^{a b}\left(\nabla_{a} f(t)\right)\left(\nabla_{b} f(t)\right)\right] .
\end{aligned}
$$

Because $\nabla_{a} f(t)=f^{\prime}(t) \nabla_{a} t$ and $\nabla_{a} \nabla_{b} f(t)=f^{\prime \prime}(t)\left(\nabla_{a} t\right)\left(\nabla_{b} t\right)$, we can use the fact that $\eta^{a b}\left(\nabla_{a} t\right)\left(\nabla_{b} t\right)=-1$ to simplify.

$$
\begin{aligned}
R_{a b} & =\left(2 f^{\prime}(t)^{2}-2 f^{\prime \prime}(t)\right)\left(\nabla_{a} t\right)\left(\nabla_{b} t\right)+\left(2 f^{\prime}(t)^{2}+f^{\prime \prime}(t)\right) \eta_{a b} \\
R & =\Omega^{-2}\left[6 f^{\prime \prime}(t)+6 f^{\prime}(t)^{2}\right] .
\end{aligned}
$$

Using Einstein's equation $R_{a b}-\frac{1}{2} R g_{a b}=8 \pi T_{a b}$ we have the following.

$$
T_{a b}=\frac{1}{8 \pi}\left[\left(2 f^{\prime}(t)^{2}-2 f^{\prime \prime}(t)\right)\left(\nabla_{a} t\right)\left(\nabla_{b} t\right)-\left(f^{\prime}(t)^{2}+2 f^{\prime \prime}(t)\right) \eta_{a b}\right]
$$

Letting $\rho=3 f^{\prime}(t)^{2} / 8 \pi$ and $p=-\left(f^{\prime}(t)^{2}+2 f^{\prime \prime}(t)\right) / 8 \pi$, we see that $T_{a b}=$ $(\rho+p) \nabla_{a} t \nabla_{b} t+p \eta_{a b}$ as claimed.

Proposition 4. $\left(^{*}\right)$ is false for all $\mathscr{P}$ such that $(D E C) \subset \mathscr{P} \subset(N E C)$.

Proof. Let $\mathscr{P}$ be such that $(D E C) \subset \mathscr{P} \subset(N E C)$. We are done if we can construct an extendible model in the collection $(D E C)$ which has no extension in the collection $(N E C)$. Consider Minkowski spacetime $\left(\mathbb{R}^{4}, \eta_{a b}\right)$ where $\eta_{a b}=$ $-\nabla_{a} t \nabla_{b} t+\nabla_{a} x \nabla_{b} x+\nabla_{a} y \nabla_{b} y+\nabla_{a} z \nabla_{b} z$. Let $\Omega: \mathbb{R}^{4} \rightarrow \mathbb{R}$ be defined by $\Omega(t, x, y, z)=\exp [f(t)]$ where $f(t)=t^{2} / 2+t$. By the first lemma, the energymomentum tensor associated with $g_{a b}=\Omega^{2} \eta_{a b}$ is given by

$$
T_{a b}=(\rho+p) \nabla_{a} t \nabla_{b} t+p \eta_{a b}
$$

where $\rho=3 f^{\prime}(t)^{2} / 8 \pi=3(t+1)^{2} / 8 \pi$ and $p=-\left(f^{\prime}(t)^{2}+2 f^{\prime \prime}(t)\right) / 8 \pi=-((t+$ $\left.1)^{2}+2\right) / 8 \pi$. So we have the following.

$$
\rho-|p|=\rho+p=\left(2(t+1)^{2}-2\right) / 8 \pi
$$

We see that if $t>0$, then $\rho-|p|>0$. Consider the extendible model $\left(M, g_{a b}\right)$ where $M=\left\{(t, x, y, z) \in \mathbb{R}^{4}: t>0\right\}$. Since $\rho-|p|>0$ on $M$, it follows that the 
model is in the collection $(D E C)$ (Wald 1984, 220). We need only show that any extension to $\left(M, g_{a b}\right)$ is not in the collection $(N E C)$.

Let $\left(M^{\prime}, g_{a b}^{\prime}\right)$ be any extension to $\left(M, g_{a b}\right)$ and let $q \in M^{\prime}$ be a point in the boundary of $M$. Let $(O, \varphi)$ be a chart with $q \in O$ such that we can extend the coordinates $(t, x, y, z)$ on $M$ to $M \cup O$. Let $q_{1}, q_{2}, q_{3} \in \mathbb{R}$ be such that $q=\left(0, q_{1}, q_{2}, q_{3}\right)$. Consider any smooth functions $\alpha_{t}, \alpha_{x}, \alpha_{y}, \alpha_{z}: O \rightarrow \mathbb{R}$ such that

$$
\chi^{a}=\alpha_{t}(\partial / \partial t)^{a}+\alpha_{x}(\partial / \partial x)^{a}+\alpha_{y}(\partial / \partial y)^{a}+\alpha_{z}(\partial / \partial z)^{a}
$$

is a null vector field on $O$ and we have $\alpha_{t}=\alpha_{x}=1$ and $\alpha_{y}=\alpha_{z}=0$ on $O \cap M$. Now find some $\delta>0$ such that $\left(-\delta, q_{1}, q_{2}, q_{3}\right) \in O$. For $t \in(-\delta, \delta)$, let $q(t)=\left(t, q_{1}, q_{2}, q_{3}\right) \in M^{\prime}$. Consider the smooth function $h:(-\delta, \delta) \rightarrow \mathbb{R}$ given by $h(t)=T_{a b}^{\prime} \chi^{a} \chi_{\mid q(t)}^{b}$ where $T_{a b}^{\prime}$ is defined on $M^{\prime}$ in the natural way (using the metric $g_{a b}^{\prime}$ and Einstein's equation). Of course, for all $t>0$, we have

$$
\begin{aligned}
h(t) & =T_{a b}^{\prime} \chi^{a} \chi_{\mid q(t)}^{b}=T_{a b} \chi^{a} \chi_{\mid q(t)}^{b}=(\rho+p)_{\mid q(t)} \\
& =\left(2(t+1)^{2}-2\right) / 8 \pi=\left(2 t^{2}+4 t\right) / 8 \pi
\end{aligned}
$$

Smoothness requires $h(0)=0$ and $h^{\prime}(0)=1 / 2 \pi$. This allows us to find an $\epsilon \in(-\delta, 0)$ such that $h(t)<0$ for $t \in(\epsilon, 0)$. So the null energy condition is violated at $q(t)$ for all $t \in(\epsilon, 0)$.

Proposition 4. $\left(^{*}\right)$ is false for all $\mathscr{P}$ such that $(S E C) \subset \mathscr{P} \subset(N E C)$.

Proof. The argument follows the one above very closely. Let $\mathscr{P}$ be such that $(S E C) \subset \mathscr{P} \subset(N E C)$. We are done if we can construct an extendible model in the collection $(S E C)$ which has no extension in the collection $(N E C)$. Consider Minkowski spacetime $\left(\mathbb{R}^{4}, \eta_{a b}\right)$ where $\eta_{a b}=-\nabla_{a} t \nabla_{b} t+\nabla_{a} x \nabla_{b} x+\nabla_{a} y \nabla_{b} y+$ $\nabla_{a} z \nabla_{b} z$. Let $\Omega: \mathbb{R}^{4} \rightarrow \mathbb{R}$ be defined by $\Omega(t, x, y, z)=\exp [f(t)]$ where $f(t)=$ $-t^{3} / 3$. By the first lemma, the energy-momentum tensor associated with $g_{a b}=$ $\Omega^{2} \eta_{a b}$ is given by

$$
T_{a b}=(\rho+p) \nabla_{a} t \nabla_{b} t+p \eta_{a b}
$$

where $\rho=3 f^{\prime}(t)^{2} / 8 \pi=3 t^{4} / 8 \pi$ and $p=-\left(f^{\prime}(t)^{2}+2 f^{\prime \prime}(t)\right) / 8 \pi=\left(4 t-t^{4}\right) / 8 \pi$. So we have the following.

$$
\begin{aligned}
\rho+p & =\left(2 t^{4}+4 t\right) / 8 \pi \\
\rho+3 p & =12 t / 8 \pi
\end{aligned}
$$

We see that if $t>0$, then both $\rho+p>0$ and $\rho+3 p>0$. Consider the extendible model $\left(M, g_{a b}\right)$ where $M=\left\{(t, x, y, z) \in \mathbb{R}^{4}: t>0\right\}$. Since $\rho+p>0$ and $\rho+3 p>0$ on $M$, it follows that the model is in the collection (SEC) (Wald $1984,220)$. We need only show that any extension to $\left(M, g_{a b}\right)$ is not in the collection $(N E C)$. 
Let $\left(M^{\prime}, g_{a b}^{\prime}\right)$ be any extension to $\left(M, g_{a b}\right)$ and let $q \in M^{\prime}$ be a point in the boundary of $M$. Let $(O, \varphi)$ be a chart with $q \in O$ such that we can extend the coordinates $(t, x, y, z)$ on $M$ to $M \cup O$. Let $q_{1}, q_{2}, q_{3} \in \mathbb{R}$ be such that $q=\left(0, q_{1}, q_{2}, q_{3}\right)$. Consider any smooth functions $\alpha_{t}, \alpha_{x}, \alpha_{y}, \alpha_{z}: O \rightarrow \mathbb{R}$ such that

$$
\chi^{a}=\alpha_{t}(\partial / \partial t)^{a}+\alpha_{x}(\partial / \partial x)^{a}+\alpha_{y}(\partial / \partial y)^{a}+\alpha_{z}(\partial / \partial z)^{a}
$$

is a null vector field on $O$ and we have $\alpha_{t}=\alpha_{x}=1$ and $\alpha_{y}=\alpha_{z}=0$ on $O \cap M$. Now find some $\delta>0$ such that $\left(-\delta, q_{1}, q_{2}, q_{3}\right) \in O$. For $t \in(-\delta, \delta)$, let $q(t)=\left(t, q_{1}, q_{2}, q_{3}\right) \in M^{\prime}$. Consider the smooth function $h:(-\delta, \delta) \rightarrow \mathbb{R}$ given by $h(t)=T_{a b}^{\prime} \chi^{a} \chi_{\mid q(t)}^{b}$ where $T_{a b}^{\prime}$ is defined on $M^{\prime}$ in the natural way (using the metric $g_{a b}^{\prime}$ and Einstein's equation). Of course, for all $t>0$, we have

$$
h(t)=T_{a b}^{\prime} \chi^{a} \chi_{\mid q(t)}^{b}=T_{a b} \chi^{a} \chi_{\mid q(t)}^{b}=(\rho+p)_{\mid q(t)}=\left(2 t^{4}+4 t\right) / 8 \pi
$$

Smoothness requires $h(0)=0$ and $h^{\prime}(0)=1 / 2 \pi$. This allows us to find an $\epsilon \in(-\delta, 0)$ such that $h(t)<0$ for $t \in(\epsilon, 0)$. So the null energy condition is violated at $q(t)$ for all $t \in(\epsilon, 0)$.

Lemma 2. Let $\mathfrak{F}(\mathscr{U})$ be the collection of framed models. Let $\leq$ denote the relation on $\mathfrak{F}(\mathscr{U})$ such that $\left(M, g_{a b}, F\right) \leq\left(M^{\prime}, g_{a b}^{\prime}, F^{\prime}\right)$ iff $\left(M^{\prime}, g_{a b}^{\prime}, F^{\prime}\right)$ is a framed extension of $\left(M, g_{a b}, F\right)$. The relation $\leq$ is a partial ordering on $\mathscr{F}(\mathscr{U})$.

Proof. See Geroch (1969, 188-189).

Lemma 3. Let $\mathfrak{F}(\mathscr{L})$ be the collection of framed models satisfying any local property $\mathscr{L} \subset \mathscr{U} \cdot \mathfrak{F}(\mathscr{L})$ is partially ordered by $\leq$ and every sub-collection of $\mathfrak{F}(\mathscr{L})$ which is totally ordered by $\leq$ has an upper bound.

Proof. Let $\mathfrak{F}(\mathscr{L})$ be the collection of framed models satisfying any local property $\mathscr{L} \subset \mathscr{U}$. Since $\mathfrak{F}(\mathscr{L}) \subset \mathfrak{F}(\mathscr{U})$, it follows from Lemma 2 that $\mathfrak{F}(\mathscr{L})$ is partially ordered by $\leq$. Let $\left\{\left(M_{i}, g_{i}, F_{i}\right)\right\}$ be a sub-collection of $\mathfrak{F}(\mathscr{L})$ which is totally ordered by $\leq$. Following Hawking and Ellis $(1973,249)$, let $M$ be the union of all the $M_{i}$ where, for $\left(M_{i}, g_{i}, F_{i}\right) \leq\left(M_{j}, g_{j}, F_{j}\right)$, each $p_{i} \in M_{i}$ is identified with $\varphi_{i j}\left(p_{i}\right)$ where $\varphi_{i j}: M_{i} \rightarrow M_{j}$ is the unique isometric embedding which takes $F_{i}$ into $F_{j}$. The manifold $M$ will have an induced metric $g=\varphi_{i *} g_{i}$ on each $\varphi_{i}\left[M_{i}\right]$ where $\varphi_{i}: M_{i} \rightarrow M$ is the natural isometric embedding. Finally, take $F$ to be the result of carrying along a chosen $F_{i}$ using $\varphi_{i}: M_{i} \rightarrow M$. Consider the framed model $(M, g, F)$. Clearly, for all $i$, we have $\left(M_{i}, g_{i}, F_{i}\right) \leq(M, g, F)$. Consider the open cover $\left\{M_{i}\right\}$ on M. Since each $\left(M_{i}, g_{i}, F_{i}\right) \in \mathfrak{F}(\mathscr{L})$, it follows from the fact that $\mathscr{L}$ is local that $(M, g, F) \in \mathfrak{F}(\mathscr{L})$. We see that $(M, g, F)$ is an upper bound for $\left\{\left(M_{i}, g_{i}, F_{i}\right)\right\}$.

Proposition 6. $\left({ }^{* *}\right)$ is true for all local $\mathscr{P} \subset \mathscr{U}$. 
Proof. Let $\mathscr{L} \subset \mathscr{U}$ be a local property and let $\left(M, g_{a b}\right)$ be an $\mathscr{L}$-model which is $\mathscr{L}$-extendible. Let $F$ be frame at some point $p \in M$. So, $\left(M, g_{a b}, F\right) \in \mathfrak{F}(\mathscr{L})$. By Lemma 3 and Zorn's lemma, there is a maximal element $\left(M^{\prime}, g_{a b}^{\prime}, F^{\prime}\right) \in$ $\mathfrak{F}(\mathscr{L})$ such that $\left(M, g_{a b}, F\right) \leq\left(M^{\prime}, g_{a b}^{\prime}, F^{\prime}\right)$. It follows that $\left(M^{\prime}, g_{a b}^{\prime}\right)$ is a $\mathscr{L}$ inextendible $\mathscr{L}$-extension of $\left(M, g_{a b}\right)$.

Proposition 8. ${ }^{(*)}$ is true if $\mathscr{P}=(G I)$.

Proof. Let $\left(M, g_{a b}\right)$ be a geodesically incomplete model which has a geodesically incomplete extension - call this extension $\left(M^{\prime}, g^{\prime}\right)$. If $\left(M^{\prime}, g^{\prime}\right)$ is inextendible, we are done. Suppose $\left(M^{\prime}, g^{\prime}\right)$ is extendible and let $\left(M^{\prime \prime}, g^{\prime \prime}\right)$ be any inextendible extension to $\left(M^{\prime}, g_{a b}^{\prime}\right)$. If $\left(M^{\prime \prime}, g_{a b}^{\prime \prime}\right)$ is geodesically incomplete, we are done. Suppose $\left(M^{\prime \prime}, g_{a b}^{\prime \prime}\right)$ is geodesically complete. The model $\left(M^{\prime \prime}-\{p\}, g_{a b}^{\prime \prime}\right)$ for $p \in M^{\prime \prime}-M^{\prime}$ is extendible and therefore geodesically incomplete. By construction, $M$ is a proper subset of $M^{\prime \prime}-\{p\}$ and so $\left(M^{\prime \prime}-\{p\}, g_{a b}^{\prime \prime}\right)$ is an extension of $\left(M, g_{a b}\right)$. But $\left(M^{\prime \prime}-\{p\}, g_{a b}^{\prime \prime}\right)$ has only one extension: the geodesically complete $\left(M^{\prime \prime}, g_{a b}^{\prime \prime}\right)$.

\section{References}

[1] Andréka, H., Németi, I., Madarász, J., and Székely, G. (2010), "On Logical Analysis of Relativity Theories," Hungarian Philosophical Review, 4: 204-222.

[2] Ashtekar, A. (2010), "The Big Bang and the Quantum," AIP Conference Proceedings, 1241: 109.

[3] Bernal, A., and Sánchez, M. (2007), "Globally Hyperbolic Spacetimes Can Be Defined as 'Causal' Instead of 'Strongly Causal," Classical and Quantum Gravity, 24: 745-749.

[4] Chrusciel, P., and Isenberg, J. (1993). "Nonisometric Vacuum Extensions of Vacuum Maximal Globally Hyperbolic Spacetimes," Physical Review D 48: 1616.

[5] Clarke, C. (1976), "Spacetime Singularities," Communications in Mathematical Physics, 49: 17-23.

[6] Clarke, C. (1993), The Analysis of Space-Time Singularities. Cambridge: Cambridge University Press.

[7] Curiel, E. (2017): "A Primer on Energy Conditions," in D. Lehmkuhl, G. Schiemann, and E. Scholz (eds.), Towards a Theory of Spacetime Theories, Boston: Birkhäuser, 43-104.

[8] Earman, J. (1995). Bangs, Crunches, Whimpers, and Shrieks: Singularities and Acausalities in Relativistic Spacetimes. Oxford: Oxford University Press. 
[9] Earman, J., Wüthrich, C., and Manchak, J. (2016). "Time Machines", in E. Zalta (ed.), Stanford Encyclopedia of Philosophy, <plato.stanford.edu/ archives/win2016/entries/time-machine/>.

[10] Ellis G. and B. Schmidt (1977). "Singular Space-Times," General Relativity and Gravitation, 8: 915-953.

[11] Galloway, G. and Ling, E. (2017) "Some Remarks on the $C^{0}$ (In)Extendibility of Spacetimes." Annales Henri Poincaré, 18: 3427-3447.

[12] Geroch, R. (1968), "What Is a Singularity in General Relativity?" Annals of Physics, 48: 526-540.

[13] Geroch, R. (1969), "Limits of Spacetimes," Communications in Mathematical Physics, 13: 180-193.

[14] Geroch, R. (1970), "Singularities," in M. Carmeli, S. Fickler, and L. Witten (eds.), Relativity. New York: Plenum Press, 259-291.

[15] Geroch, R. (1971), "Spacetime Structure from a Global Viewpoint", in B. K. Sachs (ed.), General Relativity and Cosmology. New York: Academic Press, 71-103.

[16] Geroch, R. (1977), "Prediction in General Relativity", in J. Earman, C. Glymour, and J. Stachel (eds.), Foundations of Space-Time Theories. Minnesota Studies in the Philosophy of Science, vol. 8. Minneapolis: University of Minnesota Press, 81-93.

[17] Geroch, R., and Horowitz, G. (1979). "Global Structure of Spacetimes," in S. W. Hawking and W. Israel (eds), General Relativity: An Einstein Centenary Survey. Cambridge: Cambridge University Press, 212-293.

[18] Hawking, S. (1969). "The Existence of Cosmic Time Functions." Proceedings of the Royal Society A. 308: 433-435.

[19] Hawking, S., and Penrose, R. (1970). "The Singularities of Gravitational Collapse and Cosmology," Proceedings of the Royal Society A, 314:529-548.

[20] Hawking, S., and Ellis, G. (1973), The Large Scale Structure of Space-Time. Cambridge: Cambridge University Press.

[21] Krasnikov, S. (2014), "Corrigendum: No Time Machines in Classical General Relativity," Classical and Quantum Gravity, 31: 079503.

[22] Krasnikov, S. (2018), Back-in-Time and Faster-than-Light Travel in General Relativity. Springer International Publishing.

[23] Low, R. (2012). "Time Machines, Maximal Extensions and Zorn's Lemma." Classical and Quantum Gravity, 29: 097001. 
[24] Malament, D. (2008). "Norton's Slippery Slope," Philosophy of Science, 75: 799-816.

[25] Malament, D. (2012). Topics in the Foundations of General Relativity and Newtonian Gravitation Theory. Chicago: University of Chicago Press.

[26] Manchak, J. (2009). "Can We Know the Global Structure of Spacetime?" Studies in History and Philosophy of Modern Physics, 40: 53-56.

[27] Manchak, J. (2011). "What is a Physically Reasonable Spacetime?" Philosophy of Science, 78: 410-420.

[28] Manchak, J. (2013). "Global Spacetime Structure." The Oxford Handbook of Philosophy of Physics, R. Batterman (ed.), 587-606. Oxford: Oxford University Press.

[29] Manchak, J. (2014) "On Space-Time Singularities, Holes, and Extensions." Philosophy of Science 81: 1066-1076.

[30] Manchak, J. (2016). "Is the Universe As Large as It Can Be?" Erkenntnis, 81: 1341-1344.

[31] Manchak, J. (2017). "On the Inextendibility of Space-Time." Philosophy of Science, 84: 1215-1225.

[32] Minguzzi, E. (2012). "Causally Simple Inextendible Spacetimes Are HoleFree." Journal of Mathematical Physics, 53: 062501.

[33] Norton, J. (2003), "Causation as Folk Science," Philosophers' Imprint, 3: $1-22$.

[34] Penrose, R. (1979). "Singularities and Time-Asymmery," in S. Hawking and W. Israel (eds), General Relativity: An Einstein Centenary Survey. Cambridge: Cambridge University Press, 581-638.

[35] Sbierski, J. (2018). "On the Proof of the $C^{0}$-inextendibility of the Schwarzschild Spacetime." Journal of Physics: Conference Series, 968: 012012 .

[36] Wald, R. (1984). General Relativity. Chicago: University of Chicago Press.

[37] Weatherall, J. (2014). "What Is a Singularity in Geometrized Newtonian Gravitation?" Philosophy of Science, 81: 1077-1089. 